Published in final edited form as:

Magn Reson Med. 2019 May ; 81(5): 3153-3167. doi:10.1002/mrm.27647.

\title{
Efficient shear wave elastography using transient acoustic radiation force excitations and MR displacement encoding
}

\author{
Lorne W. Hofstetter ${ }^{1}$, Henrik Odéen ${ }^{1}$, Bradley D. Bolster Jr. ${ }^{2}$, Alexander Mueller ${ }^{1}$, Douglas \\ Christensen $^{3,4}$, Allison Payne ${ }^{1}$, and Dennis L. Parker ${ }^{1}$ \\ ${ }^{1}$ Department of Radiology and Imaging Sciences, University of Utah, Salt Lake City, Utah, USA \\ 2Siemens Medical Solutions USA, Inc., Salt Lake City, Utah, USA \\ ${ }^{3}$ Department of Bioengineering, University of Utah, Salt Lake City, Utah, USA \\ ${ }^{4}$ Department of Electrical and Computer Engineering, University of Utah, Salt Lake City, Utah, \\ USA
}

\begin{abstract}
Purpose: To present a novel magnetic resonance shear wave elastography (MR-SWE) method that efficiently measures the speed of propagating wave packets generated using acoustic radiation force (ARF) impulses.
\end{abstract}

Methods: ARF impulses from a focused ultrasound (FUS) transducer were applied sequentially to a preselected set of positions and motion encoded MRI was used to acquire volumetric images of the propagating shear wavefront emanating from each point. The wavefront position at multiple propagation times was encoded in the MR phase image using a train of motion encoding gradient lobes. Generating a transient propagating wavefront at multiple spatial positions and sampling each at multiple time-points allowed for shear wave speed maps to be efficiently created. MRSWE was evaluated in tissue mimicking phantoms and ex vivo bovine liver tissue before and after ablation.

Results: MR-SWE maps, covering an in-plane area of $\sim 5 \times 5 \mathrm{~cm}$, were acquired in 12 seconds for a single slice and 144 seconds for a volumetric scan. MR-SWE detected inclusions of differing stiffness in a phantom experiment. In bovine liver, mean shear wave speed significantly increased from $1.65 \pm 0.18 \mathrm{~m} / \mathrm{s}$ in normal to $2.52 \pm 0.18 \mathrm{~m} / \mathrm{s}$ in ablated region ( $\mathrm{n}=581$ pixels, $\mathrm{p}$-value $<0.001$ ).

Conclusion: MR-SWE is an elastography technique that enables precise targeting and excitation of the desired tissue of interest. MR-SWE may be particularly well suited for treatment planning and endpoint assessment of MR-guided FUS procedures since the same device used for therapy can be used as an excitation source for tissue stiffness quantification.

\section{Keywords}

elastography; acoustic radiation force; shear wave speed; MRE; ARFI

Corresponding Author: Lorne Hofstetter, INC, 729 Arapeen Drive, Salt Lake City, UT 84108, Phone: 208-860-8609, lorne.hofstetter@hsc.utah.edu. 


\section{Introduction}

Clinicians use manual palpation to assess tissue stiffness and to evaluate and detect pathology. While palpation is widely used as part of the physical diagnosis, it is qualitative and generally limited to superficial regions. Therefore, researchers are developing and evaluating quantitative imaging approaches to non-invasively measure stiffness in both superficial and deep seated tissues.

A variety of ultrasound (1-4) and MR (5-12) elastography methods have been developed to measure the shear wave speed $\left(c_{s}\right)$ or shear modulus $(\mu)$ of tissues. In an ideal elastic nondissipative medium, the speed of a propagating shear wave is related to the shear modulus by:

$$
\mu=\rho c_{s}^{2}
$$

where $\rho$ is the medium density $(13,14)$. The shear modulus provides a quantitative representation of the tissue properties that are qualitatively evaluated using manual palpation (15).

In many elastography techniques, a stress is applied to the tissue and the resulting dynamic tissue strain is monitored with imaging. In MR elastography (MRE), the induced mechanical strain is encoded in the MR image phase using motion-encoding gradients (MEGs). Mechanical excitation is typically achieved using vibratory motion from an external oscillatory driver or the acoustic radiation force (ARF) from a focused ultrasound (FUS) transducer (16). The use of either transient $(6,9-12)$ or harmonic (i.e., steady-state) $(5,7,8)$ excitation modes have been explored. Harmonic MRE techniques are the most developed and have been used to detect liver fibrosis (17) and measure tissue mechanical properties in brain, breast, kidney, and heart $(16,18)$. Elastography techniques that utilize the acoustic radiation force for mechanical excitation may be advantageous for certain applications. With a FUS transducer, it is possible to supply the mechanical excitation directly into the tissue of interest. Additionally, the predominant direction of the propagating shear wave is perpendicular to the FUS beam (3), which makes orientation of the MEG straightforward. Finally, FUS induced MRE could become an important tool for treatment planning, monitoring, and endpoint assessment in MR-guided focused ultrasound therapies. A main benefit here being that the same focused ultrasound device used for treatment can be used for elastography.

The idea of using MR to measure the shear wave speed from an ARF generated wave packet was first demonstrated by Sarvazyan et al. (6). By varying the time between the ARF impulse and MEG, the position of the transient shear wave packet was measured in a phantom for different propagation times. Souchon et al. showed that measuring transient shear waves in ex vivo biological tissues was also feasible (11). Using a small bore 7 Tesla scanner and large amplitude MEGs (140mT/m), Liu et al. demonstrated that 2D shear wave speed maps can be computed from these MR measured transient shear waves $(9,12)$.

Potential challenges to implementing this method clinically include the long acquisition time 
( $\sim 30$ minutes for a 2D map) and use of MEG amplitudes that are not currently available on clinical whole-body MRI systems.

The purpose of this study was to develop a rapid MRI-based method of generating both 2D and volumetric 3D shear wave speed maps from ARF generated impulses. To improve acquisition efficiency, an MEG train following a single ARF impulse is used to encode the location of the propagating shear wave packet at multiple time-points in each phase image. Leveraging the electronic steering capabilities of phased array FUS transducers and using an interleaving scheme described previously $(19,20)$, phase images are acquired such that the ARF impulse spatial position for each image is different. The benefit of this multi-point approach is three-fold: (1) ARF induced shear wavefronts attenuate rapidly which limits the spatial extent of the region over which a shear wave speed can be measured. By varying the ARF location, shear wave speeds over a larger volume can be achieved when measurements are combined together. (2) From overlapped regions, the propagation time between the initial ARF impulse and the next encoded wavefront can be determined. This allows the data to be more fully utilized - shear wave speed between initial ARF location and the first encoded wavefront can be computed if this timing is known. (3) The redundant overlapping measurements can be combined to improve the precision of the measured shear wave speed. Both the efficient shear wavefront encoding scheme and acquisition of images with different ARF impulse locations generate a very rich dataset from which high-resolution shear wave speed maps can be generated. Using this new magnetic resonance shear wave elastography (MR-SWE) technique complete acquisition of a 2D map is possible within a 12 second breath-hold.

In this study we describe the MR-SWE acquisition and automated algorithm used to calculate shear wave speed maps. MR-SWE was evaluated in tissue mimicking phantoms and compared to a conventional harmonic MRE technique. The feasibility of using MRSWE for treatment assessment was demonstrated in an ex vivo bovine liver ablation experiment.

\section{Methods}

\section{Pulse Sequence}

For the MR-SWE acquisition (Figure 1), four MEG lobes oriented parallel to the FUS beam were added to a 3D gradient echo segmented echo planar imaging (EPI) pulse sequence. Optical triggers synchronized the short ARF impulses to start a time $\tau$ before the start of the first MEG lobe. If the shape and amplitude of the ARF generated wave packet does not change significantly over the duration of each MEG lobe, the four lobes sample the cylindrically propagating shear wavefront at times $\tau+\Delta t / 2, \tau+3 \Delta t / 2, \tau+5 \Delta t / 2$, and $\tau+7 \Delta t / 2$ where $\Delta t$ is the center-to-center spacing between MEG lobes. The parameter $\tau$ was chosen such that the initial ARF excitation position was encoded as a point location. ARF impulses located at multiple spatial positions were interleaved on a repetition time (TR) level and a complete image volume was acquired for each ARF location. The use of a FUS phased array transducer enabled rapid switching of the ARF spatial position. For the last interleaved TR, an interleaved off image (i.e., no ARF disturbance) was acquired as a reference. The acquisition was repeated until k-space was fully sampled such that a complete imaging 
volume was acquired for each ARF position and the reference. The reference image was subtracted from all ARF encoded phase images to remove any unwanted background or temperature induced phase change.

\section{Shear Wave Speed from MR Phase Images}

ARF generated mechanical shear waves in an infinite viscoelastic soft solid can be modeled using the Green's Function method by Bercoff et al. (21). Using the pure shear component of this model, displacement along the y-direction generated by a cylindrically symmetric forcing function along y can be calculated using the following expression:

$$
u(\vec{r}, t)=f(\vec{r}, t) \otimes g_{s}(\vec{r}, t)
$$

where, $\vec{r}$ is the radial position, $r=|\vec{r}|, f(\vec{r}, t)$ is the ARF generated forcing function along the y direction, $\otimes$ denotes the 2D convolution in both $\vec{r}$ and time $t$, and,

$$
g_{s}(\vec{r}, t)=\frac{1}{4 \pi \rho c_{s}} \frac{1}{\sqrt{2 \pi \nu_{s} t}} \frac{r^{2}-y^{2}}{r^{3}} e^{-\frac{\left(c_{s} t-r\right)^{2}}{2 v_{s} t}}
$$

is the shear component of the Green's Function where $\rho$ is the density, $c_{s}$ is the shear wave speed, and $v_{s}$ is the kinematic shear viscosity of the media. Accrued MR phase, at time $t$, from these mechanical shear waves can be calculated using the following expression

$$
\phi(\vec{r}, t)=\gamma \int_{0}^{t} G_{y}\left(t^{\prime}\right) \cdot u\left(\vec{r}, t^{\prime}\right) d t^{\prime}
$$

where $\gamma$ is the gyromagnetic ratio and $G_{y}(t)$ is the MEG waveform of the y-gradient. Figure 2 depicts a numerical simulation (using Equations 2-4) of an ARF induced shear wavefront and the accrued MR phase. While the shear wavefront is strictly positive, the wavefront position at each time-point is encoded as alternating positive and negative peaks in the phase image.

Figure 3a shows a graphical depiction of the positive and negative peaks in each MR phase image. If the distance between adjacent peaks and troughs can be robustly identified, a shear wave speed estimate between the $\mathrm{n}^{\text {th }}$ adjacent pair $\left(c_{s, n}\right)$ can be calculated according to: 


$$
c_{s, n}=\left\{\begin{array}{l}
\frac{\Delta r_{n}(\theta)}{\Delta t}, n \geq 1 \\
\frac{\Delta r_{n}(\theta)}{t_{0}}, n=0
\end{array}\right.
$$

where $\Delta r_{n}(\theta)$ is the distance between the $\mathrm{n}^{\text {th }}$ adjacent peak-trough pair along a radius of angle $\theta, \Delta t$ is the center-to-center spacing between MEG lobes, and $t_{0}$ is the time between the formation of the initial shear wavefront at the ARF excitation location (origin) and the next encoded shear wavefront position.

While $\Delta t$ is a parameter of the MRI pulse sequence, $t_{0}$ cannot be determined from the timing of the FUS and MRI pulse sequence alone and may depend on properties specific to the transducer and tissue being imaged. However, if multiple phase images are acquired so that the ARF locations are varied (as is shown in Figure 1 ), $t_{0}$ can be numerically estimated.

A method of estimating $t_{0}$ is outlined in Figure $3 \mathrm{~b}$ where the subscripts $j$ and $k$ are used to denote parameters associated with phase images acquired with an ARF impulse located at $\vec{R}_{j}$ and $\vec{R}_{k}$, respectively. The region where $V_{0 j}$ and $V_{1 k}$ intersect is particularly useful. In region $V_{1 k}$, the average shear wave speed along a radius of angle $\theta$ (radius shown as green line in Figure 3b) can be computed using the top expression in Equation 5. In region $V_{0 j}$, the distance between the first peak trough pair along a radius of angle $\beta$ (radius shown as magenta line in Figure 3b) can be measured. If we assume the shear wave speed is the same along these two intersecting radii segments, an estimate for $t_{0}$ at that intersection location is obtained by dividing the first peak-trough pair distance by the measured shear wave speed. A collection of estimates of $t_{0}$ can be obtained by repeating this calculation for all pixels in the region where $V_{0 j}$ and $V_{1 k}$ intersect. The overlapping region $V_{1 j}$ and $V_{0 k}$ in the Figure $3 \mathrm{~b}$ also provides an area where $t_{0}$ can be estimated. In general, each multiple-point dataset will contain many of these overlapping regions. A distribution of estimates for $t_{0}$ (denoted by $\vec{t}_{0}$ ) can be calculated from the pixels in all overlapping regions using the following expression:

$$
\vec{t}_{0}=\frac{\Delta r_{0 j}(\beta)}{c_{s, n}^{k}(\theta)}, \text { for all } j, k, \text { where } n \geq 1 \text { and } V_{0 j} \cap V_{n k}
$$

where $\Delta r_{0 j}(\beta)$ is the distance along a projection of angle $\beta$ between the ARF impulse location and the next wavefront position for the $f^{\text {th }}$ image and $c_{s, n}^{k}(\theta)$ is the calculated shear wave speed along a projection of angle $\theta$ between the $n^{\text {th }}$ peak/trough pairs of the $k^{\text {th }}$ image. 
In this work we assumed that the time between initial formation of the shear wavefront and the first encoded shear wavefront position is the same for all ARF points in a given MR-

SWE image acquisition. Thus, if multiple-points are acquired in this overlapped way, the full form of Equation 5 where $t_{0}=\operatorname{median}\left(\vec{t}_{0}\right)$ can be used to calculate shear wave speed.

\section{Composite Shear Wave Speed Map}

An overview of the MR-SWE reconstruction approach and generation of a composite shear wave speed map from multiple-point data is outlined in Figure 4. The propagating shear wavefront is visible as alternating positive and negative rings in the phase difference images (Figure 4a). Normalized 2D cross-correlation template matching with a 2D Gaussian shaped filter was used to extract the initial ARF impulse location for each ARF point. Using this location as the source of the cylindrically propagating wave, 1D template matching was used to generate an initial position estimate of the shear wavefront displacement peak along each radial line. To improve robustness of peak detection and allow sub-voxel determination of the displacement peak position, a 1D $3^{\text {rd }}$ order polynomial was fit to the local data on either side of the initial displacement peak estimate. Location of the extreme value of the fitted curve was calculated and provided the position of each shear wavefront displacement peak along each radial line (colored concentric rings in Figure 4b). With the wavefront positions identified for each ARF point, the shear wave speed for each phase image was calculated using the measured $t_{0}$ and Equation 5 (Figure $4 \mathrm{c}$ ). A composite shear wave speed map was then generated as the point-wise median combination of individual shear wave maps (Figure 4d). This median combination consisted of reporting the median value in pixels where 3 or more unique measurements of the shear wave speed existed.

To facilitate the reproducibility of our research and the presented MR-SWE reconstruction method, data and source code (Matlab) used to generate the composite shear wave speed results in this paper are available on Github (git.io/mrswe).

\section{Shear Wave Speed Measurement}

All MR-SWE measurements were performed using a 256-element, $950 \mathrm{kHz}$ frequency, FUS transducer (Imasonic, Besançon, France), with a $13-\mathrm{cm}$ focal length, $2 \times 2 \times 8 \mathrm{~mm}$ full-width at half max focal spot size, and hardware and software for electronic beam steering (Image Guided Therapy, Pessac, France). The transducer was coupled to the imaging samples with a bath of deionized and degassed water (Figure 1d - f). Custom-built single loop or 5-channel receive RF-coils were used to acquire the MR signal. MR imaging was performed using a 3 Tesla scanner (MAGNETOM Prisma ${ }^{\mathrm{FIT}}$, Siemens, Erlangen, Germany). For each MR-SWE acquisition, 16 ARF points were oriented in two concentric circles as shown by cross marks in Figure 4d. All ARF points were coplanar and contained within a radius of $8 \mathrm{~mm}$ about the geometric focus of the transducer. Duration of each MEG lobe was $3 \mathrm{~ms}$ and $4 \mathrm{~ms}$ for phantom and ex-vivo experiments, respectively. These durations were chosen empirically based on their ability to sufficiently encode displacements in MR image phase and to minimize spacing between encoded shear wavefront positions. For all measurements, motion encoding was only in one direction, selected in the direction of the FUS beam-propagation. MR data was reconstructed offline in Matlab (R2017b, The MathWorks Inc., Natick, MA, 
USA). Multi-coil data was optimally combined to generate magnitude images using Equation 24 by Roemer et al. (22) and phase difference images using Equation 4 by Parker et al. (23). All MR-SWE data was zero-filled interpolated in plane to a voxel spacing of $0.5 \times 0.5 \times 5 \mathrm{~mm}$ to minimize partial-volume effects. Except for the experiment directly comparing MR-SWE to conventional MRE, all MR-SWE measurements are reported as shear wave speed maps. Conversion to shear modulus can be made using Equation 1.

\section{Phantom Experiments}

The use of multi-lobed and single-lobed MEG encoding strategies to image ARF generated propagating wave packets was compared in a tissue-mimicking phantom. A homogeneous 125-bloom gelatin phantom (ballistics gelatin, Vyse Gelatin Co., Schiller Park, IL, USA) was constructed in an acrylic cylinder (10 cm inner diameter, $15 \mathrm{~cm}$ height) using a recipe published previously (24). The phantom was doped with 2 millimolar copper (II) sulfate pentahydrate. For the single-lobed imaging protocol, four different images were acquired such that the delay between the start of the ARF impulse and the center of the encoding MEG lobe was 3, 6, 9, and $12 \mathrm{~ms}$, respectively. This enabled the transient shear wave packet to be sampled at four different propagation times in four separate interleaved measurements. The multi-lobed protocol used a four-lobed MEG where the center-to-center spacing between adjacent lobes was $3 \mathrm{~ms}$. The polarity alternated between adjacent lobes. Timing between the start of the ARF impulse and the center of each lobe was 3,6, 9, and $12 \mathrm{~ms}$, respectively. For both multi-lobed and single-lobed measurements the following scan and FUS parameters were used: TR/TE $=47 / 29 \mathrm{~ms}$, Flip angle $=23^{\circ}$, Matrix $=128 \times 112 \times 12$, Resolution $=1 \times 1 \times 5 \mathrm{~mm}$, Bandwidth $=752 \mathrm{~Hz} /$ Pixel, Echo Train Length $=7$, MEG amplitude $=60 \mathrm{mT} / \mathrm{m}$, MEG slew rate $=100 \mathrm{~T} / \mathrm{m} / \mathrm{s} \mathrm{MEG} \mathrm{lobe} \mathrm{duration}=3 \mathrm{~ms}$, FUS duration $=3 \mathrm{~ms}$, and FUS acoustic power $=106$ Watts.

A second phantom experiment was performed to evaluate the relationship between gradient lobe spacing, intrinsic resolution of shear wave speed measurement, and the magnitude of phase accumulated for a given MEG duration. Two homogeneous gelatin phantoms with different mechanical properties (125-bloom and 62.5-bloom) were imaged using a fourlobed MEG encoding strategy following an ARF excitation. MEG lobe durations of 1.2, 2, 3, 4,5 , and $6 \mathrm{~ms}$ were tested in each phantom. Since lobes were played back-to-back, centerto-center spacing between lobes was identical to the lobe duration. For each measurement, the following scan and FUS parameters were used: TR/TE $=56 / 38 \mathrm{~ms}$, Flip angle $=25^{\circ}$, Matrix $=128 \times 112 \times 12$, Resolution $=1 \times 1 \times 5 \mathrm{~mm}$, Bandwidth $=752 \mathrm{~Hz} /$ Pixel, Echo Train Length $=7$, MEG amplitude $=60 \mathrm{mT} / \mathrm{m}$, MEG slew $100 \mathrm{~T} / \mathrm{m} / \mathrm{s}$, FUS duration $=3 \mathrm{~ms}$, and FUS acoustic power $=71$ Watts.

In tissue-mimicking phantoms, MR-SWE was evaluated and compared to conventional harmonic-excitation MRE. Two gelatin phantoms were constructed in acrylic cylinders as described above. A uniform stiffness phantom (homogeneous) was made from 125-bloom gelatin. A second phantom was constructed with two small balloon-filled inclusions where the gelatin inside each inclusion was 175-bloom (stiffer) and the gelatin surrounding the inclusions was 125-bloom (softer). For improved visualization of the inclusions on standard MR imaging, 125-bloom regions and 175-bloom regions were doped with 2 millimolar and 
5 millimolar copper (II) sulfate pentahydrate, respectively. Before the start of experiment, phantoms were allowed to equilibrate overnight to a room temperature of approximately $22{ }^{\circ} \mathrm{C}$.

Detailed MR-SWE scan parameters for the phantom experiments are shown in Table 1. MRSWE was performed using both volumetric (3D, 144 second acquisition time) and single slice (2D, 12 second acquisition time) imaging. For the volumetric imaging protocol, the shear wave speed lateral to the FUS propagation direction was measured in the 3D imaging volume. MR-SWE shear wave speed was converted to shear modulus using Equation 1 assuming the phantom had the density of water $\left(1000 \mathrm{~kg} / \mathrm{m}^{3}\right)$. Temperature change due to ARF impulses was measured using proton resonance frequency shift (PRFS) MR thermometry $(25,26)$. A baseline image was acquired prior to the start of the MR-SWE measurement. Phase subtraction of the baseline image from the MR-SWE reference image ("off" interleave) isolates the phase change due to temperature. Average field drift was measured in a region outside of the ARF deposited energy and this value was used to correct thermometry readings for field drift. For all thermometry calculations in this paper, a PRFS thermal coefficient of alpha $=-0.01 \mathrm{ppm} /{ }^{\circ} \mathrm{C}$ was used.

For comparison purposes, a steady-state harmonic MRE measurement was performed on both phantoms. A prototype 2D single-shot spin echo EPI MRE sequence was used (details in Table 1) and the mechanical excitation was generated by a pneumatic mechanical driver (Resoundant, Rochester, MN, USA). The phantom was placed on top of the passive driver and acquisitions were in the coronal plane with MEG encoding through slice. The MRE sequence was synchronized with the Resoundant configured to produce a harmonic steadystate $100 \mathrm{~Hz}$ wave excitation in the gelatin. Acquisitions were acquired over 4 phase offsets and the inversions were performed using an inline 2D multi-model direct inversion (MMDI) algorithm (27). A central slice was chosen for this measurement to coincide with the center location of the MR-SWE imaging slab. Standard flex and spine array coils (Siemens, Erlangen, Germany) were used during image acquisition.

\section{Ex Vivo Bovine Liver Experiment}

A fresh (time after death less than 12 hours) ex vivo bovine liver sample obtained from a local meat packing facility was placed in a $31{ }^{\circ} \mathrm{C}$ bath of normal saline. The sample and saline was placed under partial vacuum for 2 hours to remove any air bubbles that may have been introduced during butchering and transport of the sample. The sample was then removed from the bath and placed in a cylindrical acrylic holder. The cylinder provides support for the MR imaging coil and one end of the holder was sealed with a thin PVC membrane to hold the sample within the cylinder (setup is shown in Figure 1d). Once the sample was correctly positioned MR-SWE acquisition was performed to measure shear wave speed of the sample.

To evaluate the ability of MR-SWE to detect changes in shear wave speed due to thermal ablation, a FUS ablation was performed using the same focused ultrasound transducer used for MR-SWE. The sample was sonicated at 9 positions on a $3 \times 3$ square grid $(4 \times 4 \mathrm{~mm})$ in a plane transverse to the FUS beam direction. The FUS power supplied to each point was 43.3 acoustic watts for a duration of 21.5 seconds per point with a 5.38 second pause between 
each point. This ablation pattern was repeated twice at the exact same sonication locations. During thermal ablation a segmented EPI MR thermometry protocol (without MEGs) with the following scan parameters was used: TR/TE $=28 / 22 \mathrm{~ms}$, Flip angle $=27^{\circ}$, Matrix $=$ $128 \times 112 \times 10$, Resolution $=1 \times 1 \times 2 \mathrm{~mm}$, Bandwidth $=752 \mathrm{~Hz} /$ Pixel, Echo Train Length $=7$. Temperature maps for the ablation were reconstructed using the PRFS method. Using the temperature maps, thermal dose in cumulative equivalent minutes at $43{ }^{\circ} \mathrm{C}(\mathrm{CEM} 43)$ was calculated using previously described methods (28). By the time the sample was positioned, and imaging was localized, it was likely that the sample cooled significantly from the initial $31{ }^{\circ} \mathrm{C}$ water bath temperature. Exact sample temperature at time of ablation was not known. Room temperature $\left(2{ }^{\circ} \mathrm{C}\right)$ was used as the starting temperature for thermal dose calculations. The MR-SWE measurement was repeated after the thermal ablation and compared to pre-ablation MR-SWE measurements. All MR-SWE acquisition parameters used for the measurements are shown in Table 1.

\section{Results}

\section{Phantom Experiments}

Figure 5 compares the use of single-lobed and multi-lobed encoding strategies for imaging propagating transient shear wave packets. The single-lobed measurements (Figure 5c-5f) encode one ring (or central ARF point) per image. Increased delay times between the ARF impulse and the encoding MEG lobe resulted in an encoded displacement that was located farther from the initial ARF excitation location. Figure 5g shows imaging with the four-lobe encoding strategy. Multiple concentric rings of alternating polarity surrounding a central encoded location are visible. Addition of the four single-lobed images after alternating the sign of adjacent images (Figure 5h) generates an image that is nearly identical to the fourlobed acquisition (difference image is shown in Figure 5i with line plots comparing profiles of Figure $5 \mathrm{~g}$ and $5 \mathrm{~h}$ in Supporting Information Figure S1). These results indicate that the four-lobed encoding scheme samples the same propagation times that were imaged in singlelobed measurements.

Results from the second phantom study (Figure 6) demonstrate that longer MEG lobe durations (using a four-lobed MEG encoding scheme) result in increased phase accrual and coarser shear wave speed map resolution. For the MR-SWE algorithm, the shear wave speed of the media multiplied by the MEG spacing provides a metric for evaluating the intrinsic resolution of the multi-lobed encoding scheme. In the 125-bloom phantom, 6, 5, 4, 3, and 2 ms MEG lobe durations resulted in intrinsic resolutions of 14.6, 12.0, 9.2, 6.8, and $4.8 \mathrm{~mm}$, respectively. For the $1.2 \mathrm{~ms}$ MEG lobe duration, encoded shear wavefronts could not be resolved. In the 62.5-bloom phantom, 6, 5, 4, and 3 ms MEG durations resulted in intrinsic resolutions of 6.7, 5.5, 4.7, $3.3 \mathrm{~mm}$, respectively. For the 2 and $1.2 \mathrm{~ms}$ MEG lobe durations, encoded share wavefronts could not be easily resolved. Figure $6 \mathrm{c}$ shows that an MEG lobe spacing of $3 \mathrm{~ms}$ provides a reasonable balance between shear wave speed map resolution and phase accrual when imaging 125-bloom phantoms.

Figure 7 presents the MR-SWE measurements for homogeneous (top row) and inclusion (bottom row) phantoms. The second and third column, respectively, depict the calculated shear wave speed for 3D and 2D MR-SWE acquisitions. Both 3D and 2D acquisitions 
produced comparable composite shear wave speed maps, albeit for 2D measurement, higher power ARF impulses (106 acoustic watts instead of 71 acoustic watts) were used and phase images used to calculate the shear wave speed were noisier due to the shorter acquisition time. In the homogeneous phantom, peak temperature rise due to MR-SWE acquisition was $1.65{ }^{\circ} \mathrm{C}$ and $0.87{ }^{\circ} \mathrm{C}$ for $3 \mathrm{D}$ and $2 \mathrm{D}$ measurements, respectively. Temperature change maps for homogeneous phantom are depicted in Supporting Information Figure S2. Although 2D imaging was performed with higher power ARF impulses, measured temperature increase was still less for the 2D acquisition since the total number of ARF impulses per acquisition were significantly fewer than for the 3D acquisition. Supporting Information Figure S3 depicts the sixteen intermediate shear wave speed maps (prior to median combination) for the 3D inclusion phantom.

In Figure 7d, the border of the two inclusions can be seen by the decrease in signal intensity. Inclusion borders were traced and overlaid as black lines on the MR-SWE maps in Figure 7e and $7 f$. The regions of increased shear wave speed are well encapsulated by the traced inclusion boundary. In the homogeneous phantom, the shear wave speed mean and standard deviation $(\mu \pm \sigma)$ measured over a centrally located region of interest (ROI) was $2.06 \pm 0.04$ and $2.07 \pm 0.06 \mathrm{~m} / \mathrm{s}$ for the $3 \mathrm{D}$ and $2 \mathrm{D}$ acquisitions, respectively. In the inclusion phantom, the mean shear wave speeds of the inclusions were $2.84 \pm 0.11$ and $2.84 \pm 0.15 \mathrm{~m} / \mathrm{s}$ for $3 \mathrm{D}$ and 2D acquisitions, respectively. Mean shear wave speed of gelatin surrounding the inclusions was $2.16 \pm 0.06$ and $2.13 \pm 0.09 \mathrm{~m} / \mathrm{s}$ for $3 \mathrm{D}$ and $2 \mathrm{D}$ acquisitions, respectively, in close agreement with what was measured in the homogenous phantom.

Figure 8 compares MR-SWE and steady-state MRE shear stiffness measurements. Images compared had the same coronal slice position relative to the phantom. In the homogeneous phantom, mean shear stiffness was $4.25 \pm 0.18$ and $3.77 \pm 0.03 \mathrm{kPa}$ for MR-SWE and MRE measurements, respectively. In the inclusion phantom, average shear stiffness in the softer surrounding region was $4.65 \pm 0.25$ and $3.54 \pm 0.04 \mathrm{kPa}$ for MR-SWE and MRE measurements. The top inclusion had an average shear stiffness of $8.13 \pm 0.51$ and $7.52 \pm 0.78$ $\mathrm{kPa}$ for MR-SWE and MRE measurements, respectively. The bottom inclusion had an average shear stiffness of $7.99 \pm 0.70$ and $6.75 \pm 0.32 \mathrm{kPa}$ for MR-SWE and MRE measurements. Regions of interest used to calculated mean shear stiffness are shown in Supporting Information Figure S4. Figure 8e graphically depicts mean and standard deviation of the shear stiffness measurement in the homogeneous region and within the inclusions. For all shear stiffness measurements, the MR-SWE measurement values were larger than MRE measurements and this increase ranged from 8\% to 31\%. While the cause of this discrepancy was not determined, both methods showed relatively uniform stiffness in the homogeneous phantom and were able to delineate lesions of differing stiffness in the inclusion phantoms.

\section{Ex Vivo Experiment}

Figure 9 presents results from the ex vivo bovine liver experiment. Figure $9 a$ and $9 b$, respectively, depict the MR-SWE shear wave speed measurement before and after tissue ablation. The intermediate shear wave speed maps before median combination are depicted in Supporting Information Figures S5 and S6. The post ablation MR-SWE measurement 
shows increased shear wave speeds in the ablated region. Figure 9c shows the maximum intensity projection (MIP) of MR thermometry readings acquired during the ablation. Measured peak temperature change exceeded $80^{\circ} \mathrm{C}$. Figure $9 \mathrm{~d}$ shows the total calculated thermal dose distribution. The 240 CEM43 isodose line circumscribing the ablated region was determined and displayed as a black overlay on the shear wave speed map shown in Figure 9b. All points circumscribed by the isodose line received a dose of at least 240 CEM43. Within the thermal dose boundary in Figure $9 \mathrm{~b}(\mathrm{n}=581$ pixels), the mean shear wave speed before and after ablation was $1.65 \pm 0.18$ and $2.52 \pm 0.18 \mathrm{~m} / \mathrm{s}$, respectively. A twosample t-test showed that this shear wave speed change was statistically significant, $p$-value $<0.001$. Figure $9 \mathrm{e}-\mathrm{h}$ depicts the difference in measured shear wave speed (post ablation minus pre ablation) for 4 adjacent imaging slices. In each of the slices, the measured change in shear wave speed within the 240 CEM43 dose region was $0.42,0.64,0.87$, and $0.32 \mathrm{~m} / \mathrm{s}$ for Figure 9e - h, respectively. For the MR-SWE acquisition, the ARF impulses were coplanar and located between slices shown in Figure 9f and 9g. Slices nearest the plane of the ARF impulses and ablation sonication showed the largest change in measured shear wave speed.

\section{Discussion}

This study introduced a magnetic resonance shear wave elastography technique that measures shear wave speed of transient propagating wave packets produced by impulses from a focused ultrasound transducer. In many ways MR-SWE is an MR implementation of ultrasound (US) time-of-flight shear wave speed elastography. Time-of-flight methods use rapid US imaging (up to 10,000 frames per second) to measure the arrival time of the shear wave at specific locations $(1,3,29,30)$. The shear wave speed is then calculated from the measured arrival time and corresponding position data. While such high-speed imaging rates cannot be achieved with current MRI techniques, the MR-SWE acquisition implements a number of efficient encoding strategies to reduce acquisition time and improve spatial resolution and measurement precision. The use of a single ARF impulse followed by a MEG train, measures the wavefront position at multiple time-points in a single image. In the phantom experiments, four locations of the shear wave packet were encoded in each phase image. In ex vivo liver tissue, encoding of three locations was achieved due to more rapid attenuation of the wavefront amplitude. Acquiring and combining multiple phase images, each with distinct initial ARF impulse positions, improved spatial extent and resolution of composite shear wave speed maps. This improvement is readily apparent when the composite shear wave speed map in Figure $7 \mathrm{e}$ is compared to the 16 individual measurements from which it was generated (Supporting Information Figure S3). While the inclusion borders in any one map generated from the individual measurements are ill defined (Supporting Information Figure S3), the borders are readily visible in the composite map (Figure 7e-f). Use of a segmented-EPI acquisition and efficient interleaving of ARF points helped to minimize overall acquisition time and to spatially and temporally distribute the ARF energy deposition. Since the predominant propagation direction of ARF generated shear waves is perpendicular to FUS beam propagation direction, only one motion encoding direction was needed to encode the displacement. Using these strategies, high-resolution 
single slice shear wave speed maps were generated in 12 seconds. The use of single shot EPI, as in the traditional MRE scans, could lead to further reductions in acquisition time.

Ex vivo experiments demonstrated that MR-SWE could measure shear wave speed in bovine liver and measured speeds were within the range of clinically reported values in human liver $(16,31,32)$. Feasibility of using MR-SWE to image changes in tissue stiffness following a FUS ablation was also demonstrated. Regions of increased shear wave speed coincided with regions where thermal dose exceeded 240 CEM43 (Figure 9). The change in measured shear wave speed was most pronounced within the 240 CEM43 isodose border for the two central slices (Figure 9f and 9g) and the measured change in shear wave speed was less in the adjacent slices (Figure 9e and 9h). The reason for this could be due to the fact that FUS ablations were performed in a coplanar fashion where the plane was located between the $5 \mathrm{~mm}$ thick slices shown in Figure 9f and 9g. Thus, higher temperature and larger thermal doses were achieved in these central slices than in the surrounding regions (i.e., slices in Figure 9e, 9h).

A thermal dose threshold of 240 CEM43 is often used as a marker of complete tissue necrosis $(33,34)$. However, the relationship between tissue viability and thermal dose is nuanced and can be highly dependent on tissue type (35-37). For example, the thermal damage threshold in human skin has been reported to be as high as 600 CEM43 (37) and necrosis in brain tissues can occur at doses as low as 50 CEM43 $(33,38)$. Because of this variability, using only the thermal dose to determine the treated volume is not optimal. Elastography could complement thermal dose measurements and help improve prediction of treatment volume. An ex vivo experiment used MRE to demonstrate that stiffness of focused ultrasound ablated muscle tissue is different from that of normal muscle tissue (39). MRE has been used during laser ablation treatment of a liver tumor to measure changes in tissue stiffness following treatment (40). US elastography has also been used to measure temperature induced changes in ex vivo tissue stiffness $(41,42)$. A variety of MR acoustic radiation force imaging techniques have also been utilized to evaluate changes in ex vivo tissue stiffness following an ablation (20,43-45).

In this study we demonstrated that MR-SWE can volumetrically image changes in ex vivo tissue shear wave speed following a FUS ablation. The same FUS hardware used to perform the ablation was used to produce the mechanical excitation. Thus, repositioning of sample or use of an additional external driver was not needed to generate elastography measurement. This ability to use FUS device for both treatment and as a mechanical excitation source for elastography makes this approach particularly enticing for FUS ablative therapies.

There are several potential explanations for why the MR-SWE measured shear modulus was consistently larger than MRE readings in the phantom experiment. In the MR-SWE experiments a single rectangle impulse of fixed duration ( $3 \mathrm{~ms}$ for phantom and $4 \mathrm{~ms}$ for exvivo experiments) was used to generate a transient propagating wave packet. The central frequency of this impulse is 0 and the central lobe of the band (first zeros of the sinc function) goes from $-1 / \mathrm{T}$ to $+1 / \mathrm{T}$ where $\mathrm{T}$ is the duration of the FUS excitation. This corresponds to $\pm 333 \mathrm{~Hz}$ and $\pm 250 \mathrm{~Hz}$, respectively for the $3 \mathrm{~ms}$ and $4 \mathrm{~ms}$ excitation pulse durations. Use of Equation 1 to calculate the shear modulus from the measured group shear 
wave speed assumes a purely elastic (non-viscous) medium. However, shear wave spectroscopy measurements have shown that shear wave phase velocity increases with increasing frequency in a number of different tissues and viscous phantoms $(46,47)$. If the gelatin phantoms used in this study had viscous properties, the broadband nature of the MRSWE excitation could explain the discrepancy seen between the two measurements. Additionally, the top expression in Equation 5 assumes that the amplitude decay of the propagating wave packet is minimal over the duration of each motion encoding gradient lobe. If the amplitude varies significantly over each MEG lobe, the peak values in the MR phase image may not correspond exactly to the position of the shear wavefront at the sampling times. Another possible cause is related to the MRE acquisition used. The resolution of the MRE acquisition was lower than MR-SWE and there may have been partial volume averaging of the encoded motion. The discrepancy between MR-SWE and harmonic MRE will be investigated in future work. While the exact cause of this difference between MR-SWE and MRE measurements was not isolated in this study, the measured discrepancy in shear modulus was less than $31 \%$. MR-SWE accurately detected the location of inclusions of differing stiffness.

For all MR-SWE shear wave speed measurements, the encoded peak and trough locations in the MR image phase were used to calculate the average shear wave speed along each connecting radial segment. Using the average value along each radial ray between adjacent peaks and troughs gives rise to a streaking pattern that is visible in the individual shear wave speed maps for each ARF location (Supporting Information Figures S3b, S5b and S6b). Even after median combination the streak artifacts can still be seen in the composite shear wave speed measurements. Since every phase image contains the time-integral history of the tissue displacement, a more complete use of this encoded information (instead of just the peaks and troughs) could be used to reduce these streak artifacts, improve spatial resolution, and even measure tissue shear viscosity. Exploring MR-SWE methods that more fully utilize the encoded phase information is beyond the scope of this work but will be explored in future studies.

\section{Conclusion}

In conclusion we have introduced a new magnetic resonance shear wave elastography technique. Short ARF impulses from a FUS transducer were used to generate transient propagating shear wavefronts. The location of each wavefront, at multiple propagation times, was efficiently encoded in the MR phase image using an MEG train. This encoding scheme was implemented in an interleaved fashion so that multiple phase images, each acquired with ARF impulses at different spatial locations, were acquired. By combining this multiple-point data, high resolution maps of shear wave speed were generated. In the phantom experiment MR-SWE was capable of detecting lesions of different stiffness. Feasibility of using MR-SWE to measure changes in tissue stiffness following a FUS thermal ablation was demonstrated in the ex vivo experiment. MR-SWE is a promising elastography technique that may prove useful for treatment planning, monitoring, and endpoint assessment of focused ultrasound ablative therapies. 


\section{Supplementary Material}

Refer to Web version on PubMed Central for supplementary material.

\section{Acknowledgements}

The authors thank Rock Hadley, Robb Merrill and Emilee Minalga for designing and building the RF coils and Hailey McLean for making phantoms. This work was supported by Siemens Healthcare, the Mark H. Huntsman endowed chair, a University of Utah seed grant, and NIH grants F30CA228363, R01EB013433, R01CA172787, R01CA224141, R03EB023712, and S10OD018482.

\section{References}

1. Sandrin L, Tanter M, Catheline S, Fink M. Shear modulus imaging with 2-D transient elastography. IEEE Trans Ultrason Ferroelectr Freq Control 2002;49:426-435. [PubMed: 11989698]

2. Nightingale K, McAleavey S, Trahey G. Shear-wave generation using acoustic radiation force: in vivo and ex vivo results. Ultrasound Med Biol 2003;29:1715-1723. [PubMed: 14698339]

3. Bercoff J, Tanter M, Fink M. Supersonic shear imaging: a new technique for soft tissue elasticity mapping. IEEE Trans Ultrason Ferroelectr Freq Control 2004;51:396-409. [PubMed: 15139541]

4. Gennisson JL, Deffieux T, Fink M, Tanter M. Ultrasound elastography: principles and techniques. Diagn Interv Imaging 2013;94:487-495. [PubMed: 23619292]

5. Muthupillai R, Lomas DJ, Rossman PJ, Greenleaf JF, Manduca A, Ehman RL. Magnetic resonance elastography by direct visualization of propagating acoustic strain waves. Science 1995;269:18541857. [PubMed: 7569924]

6. Sarvazyan AP, Rudenko OV, Swanson SD, Fowlkes JB, Emelianov SY. Shear Wave Elasticity Imaging: a new ultrasonic technology of medical diagnostic. Ultrasound Med Biol 1998;24:14191435. [PubMed: 10385964]

7. Wu T, Felmlee JP, Greenleaf JF, Riederer SJ, Ehman RL. MR imaging of shear waves generated by focused ultrasound. Magn Reson Med 2000;43:111-115. [PubMed: 10642737]

8. Manduca A, Oliphant TE, Dresner MA, Mahowald JL, Kruse SA, Amromin E, Felmlee JP, Greenleaf JF, Ehman RL. Magnetic resonance elastography: non-invasive mapping of tissue elasticity. Med Image Anal 2001;5:237-254. [PubMed: 11731304]

9. Liu Y, Fite BZ, Mahakian LM, Johnson SM, Larrat B, Dumont E, Ferrara KW. Concurrent Visualization of Acoustic Radiation Force Displacement and Shear Wave Propagation with 7T MRI. PLoS One 2015;10:e0139667. [PubMed: 26439259]

10. McCracken PJ, Manduca A, Felmlee J, Ehman RL. Mechanical transient-based magnetic resonance elastography. Magn Reson Med 2005;53:628-639. [PubMed: 15723406]

11. Souchon R, Salomir R, Beuf O, Milot L, Grenier D, Lyonnet D, Chapelon JY, Rouvière O. Transient MR elastography (t-MRE) using ultrasound radiation force: theory, safety, and initial experiments in vitro. Magn Reson Med 2008;60:871-881. [PubMed: 18816871]

12. Liu Y, Liu J, Fite BZ, Foiret J, Ilovitsh A, Leach JK, Dumont E, Caskey CF, Ferrara KW. Supersonic transient magnetic resonance elastography for quantitative assessment of tissue elasticity. Phys Med Biol 2017;62:4083-4106. [PubMed: 28426437]

13. Payne A, de Bever J, Farrer A, Coats B, Parker DL, Christensen DA. A simulation technique for 3D MR-guided acoustic radiation force imaging. Med Phys 2015;42:674-684. [PubMed: 25652481]

14. Aki K, Richards PG. Quantitative Seismology. 2nd ed. Sausalito, CA: University Science Books; 2002.

15. Sarvazyan AP, Skovoroda AR, Emelianov SY, Fowlkes JB, Pipe JG, Adler RS, Buxton RB, Carson PL. Biophysical bases of elasticity imaging In: Jones JP (eds) Acoustical Imaging. Acoustical Imaging, vol 21 Springer, Boston MA; 1995.

16. Mariappan YK, Glaser KJ, Ehman RL. Magnetic resonance elastography: a review. Clin Anat 2010;23:497-511. [PubMed: 20544947] 
17. Yin M, Talwalkar JA, Glaser KJ, Manduca A, Grimm RC, Rossman PJ, Fidler JL, Ehman RL. Assessment of hepatic fibrosis with magnetic resonance elastography. Clin Gastroenterol Hepatol. 2007;5:1207-1213.e2. [PubMed: 17916548]

18. Pepin KM, Ehman RL, McGee KP. Magnetic resonance elastography (MRE) in cancer: technique, analysis, and applications. Prog Nucl Magn Reson Spectrosc. 2015;90-91:32-48.

19. de Bever JT, Odéen H, Hofstetter LW, Parker DL. Simultaneous MR thermometry and acoustic radiation force imaging using interleaved acquisition. Magn Reson Med 2018;79:1515-1524. [PubMed: 28795419]

20. Odéen H, de Bever J, Hofstetter LW, Parker DL. Multiple-point magnetic resonance acoustic radiation force imaging. Magn Reson Med 2018;00:1-14.

21. Bercoff J, Tanter M, Muller M, Fink M. The role of viscosity in the impulse diffraction field of elastic waves induced by the acoustic radiation force. IEEE Trans Ultrason Ferroelectr Freq Control 2004;51:1523-1536. [PubMed: 15600098]

22. Roemer PB, Edelstein WA, Hayes CE, Souza SP, Mueller OM. The NMR phased array. Magn Reson Med 1990;16:192-225. [PubMed: 2266841]

23. Parker DL, Payne A, Todd N, Hadley JR. Phase reconstruction from multiple coil data using a virtual reference coil. Magn Reson Med 2014;72:563-569. [PubMed: 24006172]

24. Farrer AI, Odéen H, de Bever J, Coats B, Parker DL, Payne A, Christensen DA. Characterization and evaluation of tissue-mimicking gelatin phantoms for use with MRgFUS. J Ther Ultrasound 2015;3:9. [PubMed: 26146557]

25. De Poorter J, De Wagter C, De Deene Y, Thomsen C, Ståhlberg F, Achten E. Noninvasive MRI thermometry with the proton resonance frequency (PRF) method: in vivo results in human muscle. Magn Reson Med 1995;33:74-81. [PubMed: 7891538]

26. Ishihara Y, Calderon A, Watanabe H, Okamoto K, Suzuki Y, Kuroda K, Suzuki Y. A precise and fast temperature mapping using water proton chemical shift. Magn Reson Med 1995;34:814-23. [PubMed: 8598808]

27. Silva AM, Grimm RC, Glaser KJ, Fu Y, Wu T, Ehman RL, Silva AC. Magnetic resonance elastography: evaluation of new inversion algorithm and quantitative analysis method. Abdom Imaging 2015;40:810-817. [PubMed: 25742725]

28. Sapareto SA, Dewey WC. Thermal dose determination in cancer therapy. Int J Radiat Oncol Biol Phys 1984;10:787-800. [PubMed: 6547421]

29. McLaughlin J, Renzi D. Shear wave speed recovery in transient elastography and supersonic imaging using propagating fronts. Inverse Probl. 2006;22:681-706.

30. Tanter M, Bercoff J, Athanasiou A, Deffieux T, Gennisson JL, Montaldo G, Muller M, Tardivon A, Fink M. Quantitative assessment of breast lesion viscoelasticity: initial clinical results using supersonic shear imaging. Ultrasound Med Biol 2008;34:1373-1386. [PubMed: 18395961]

31. Mueller S, Sandrin L. Liver stiffness: a novel parameter for the diagnosis of liver disease. Hepat Med 2010;2:49-67. [PubMed: 24367208]

32. Cassinotto C, Lapuyade B, Mouries A, et al. Non-invasive assessment of liver fibrosis with impulse elastography: comparison of Supersonic Shear Imaging with ARFI and FibroScan®. J Hepatol 2014;61:550-557. [PubMed: 24815876]

33. Damianou C, Hynynen K. The effect of various physical parameters on the size and shape of necrosed tissue volume during ultrasound surgery. J Acoust Soc Am 1994;95:1641-1649. [PubMed: 8176064]

34. McDannold NJ, King RL, Jolesz FA, Hynynen KH. Usefulness of MR imaging-derived thermometry and dosimetry in determining the threshold for tissue damage induced by thermal surgery in rabbits. Radiology 2000;216:517-523. [PubMed: 10924580]

35. Dewhirst MW, Viglianti BL, Lora-Michiels M, Hanson M, Hoopes PJ. Basic principles of thermal dosimetry and thermal thresholds for tissue damage from hyperthermia. Int J Hyperthermia 2003;19:267-294. [PubMed: 12745972]

36. Yarmolenko PS, Moon EJ, Landon C, Manzoor A, Hochman DW, Viglianti BL, Dewhirst MW. Thresholds for thermal damage to normal tissues: an update. Int J Hyperthermia 2011;27:320-343. [PubMed: 21591897] 
37. van Rhoon GC, Samaras T, Yarmolenko PS, Dewhirst MW, Neufeld E, Kuster N. CEM $43^{\circ} \mathrm{C}$ thermal dose thresholds: a potential guide for magnetic resonance radiofrequency exposure levels? Eur Radiol 2013;23:2215-2227. [PubMed: 23553588]

38. Lyons BE, Obana WG, Borcich JK, Kleinman R, Singh D, Britt RH. Chronic histological effects of ultrasonic hyperthermia on normal feline brain Ttssue. Radiat Res 1986;106:234-2511. [PubMed: 3704114]

39. Wu T, Felmlee JP, Greenleaf JF, Riederer SJ, Ehman RL. Assessment of thermal tissue ablation with MR elastography. Magn Reson Med 2001;45:80-87. [PubMed: 11146489]

40. Chen J, Woodrum DA, Glaser KJ, Murphy MC, Gorny K, Ehman R. Assessment of in vivo laser ablation using MR elastography with an inertial driver. Magn Reson Med 2014;72:59-67. [PubMed: 23904298]

41. Sapin-de Brosses E, Gennisson J-L, Pernot M, Fink M, Tanter M. Temperature dependence of the shear modulus of soft tissues assessed by ultrasound. Phys Med Biol 2010;55:1701-1718. [PubMed: 20197599]

42. Arnal B, Pernot M, Tanter M. Monitoring of thermal therapy based on shear modulus changes: II. Shear wave imaging of thermal lesions. IEEE Trans Ultrason Ferroelectr Freq Control 2011;58:1603-1611. [PubMed: 21859579]

43. McDannold N, Maier SE. Magnetic resonance acoustic radiation force imaging. Med Phys 2008;35:3748-58. [PubMed: 18777934]

44. Bitton RR, Kaye E, Dirbas FM, Daniel BL, Pauly KB. Toward MR-guided high intensity focused ultrasound for presurgical localization: focused ultrasound lesions in cadaveric breast tissue. J Magn Reson Imaging 2012;35:1089-1097. [PubMed: 22170814]

45. Vappou J, Bour P, Marquet F, Ozenne V, Quesson B. MR-ARFI-based method for the quantitative measurement of tissue elasticity: application for monitoring HIFU therapy. Phys Med Biol 2018;63:95018.

46. Deffieux T, Montaldo G, Tanter M, Fink M. Shear wave spectroscopy for in vivo quantification of human soft tissues visco-elasticity. IEEE Trans Med Imaging 2009;28:313-322. [PubMed: 19244004]

47. Muller M, Gennisson J-L, Deffieux T, Tanter M, Fink M. Quantitative Viscoelasticity Mapping of Human Liver Using Supersonic Shear Imaging: Preliminary In Vivo Feasability Study. Ultrasound Med Biol 2009;35:219-229. [PubMed: 19081665] 

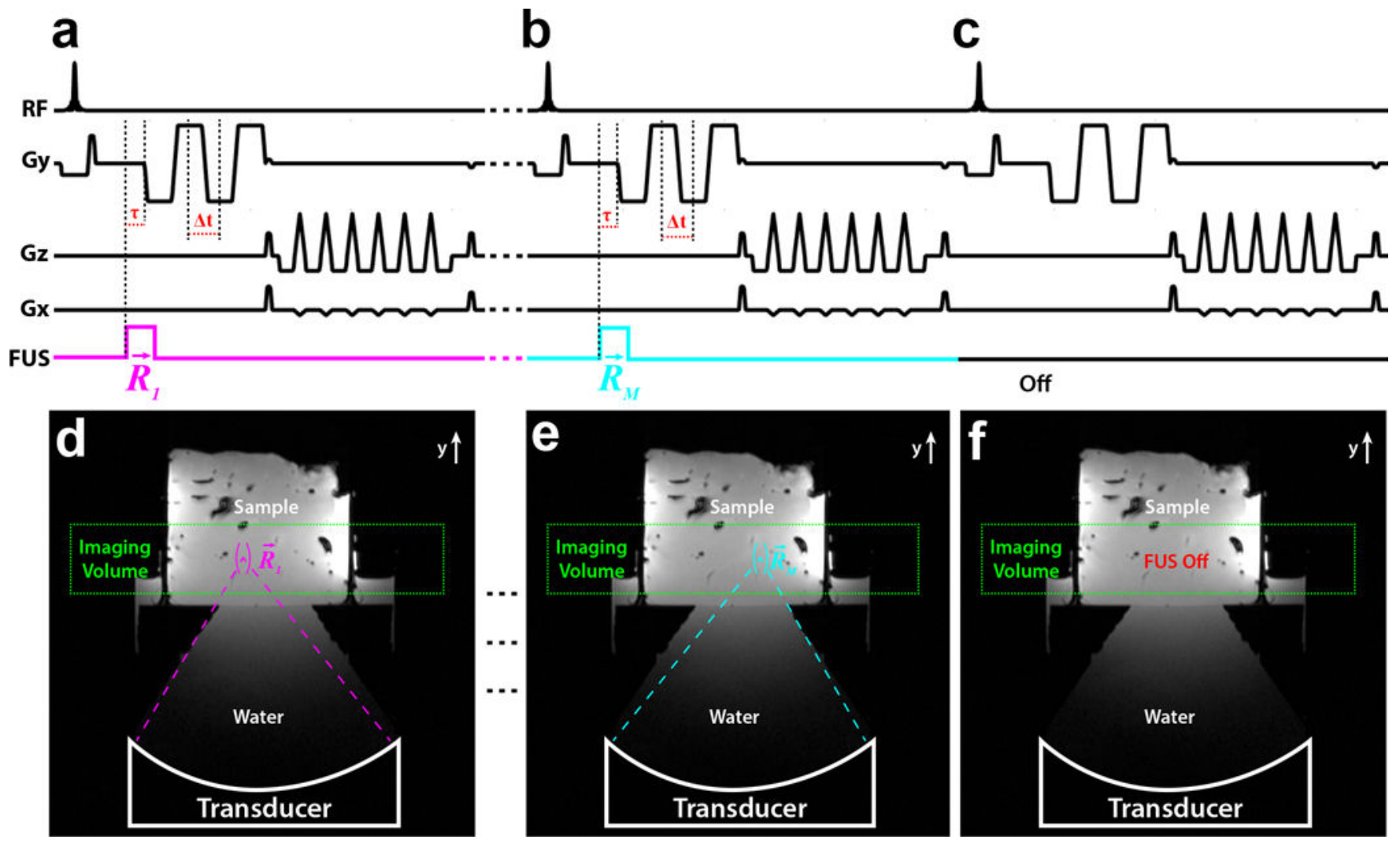

Figure 1.

Pulse sequence diagram (a-c) where differently positioned ARF impulses are interleaved on a TR level. The motion encoding gradient (MEG) encodes the initial ARF displacement at time $\tau+\Delta t / 2$ and the propagating shear wavefront at delay times of $\tau+3 \Delta t / 2, \tau+5 \Delta t / 2, \tau$ $+7 \Delta t / 2$. Experimental setup and FUS steering locations that correspond to (a-c) are shown in (d-f). The final "off image" depicted by (f) is used as the displacement free reference for all ARF images. 

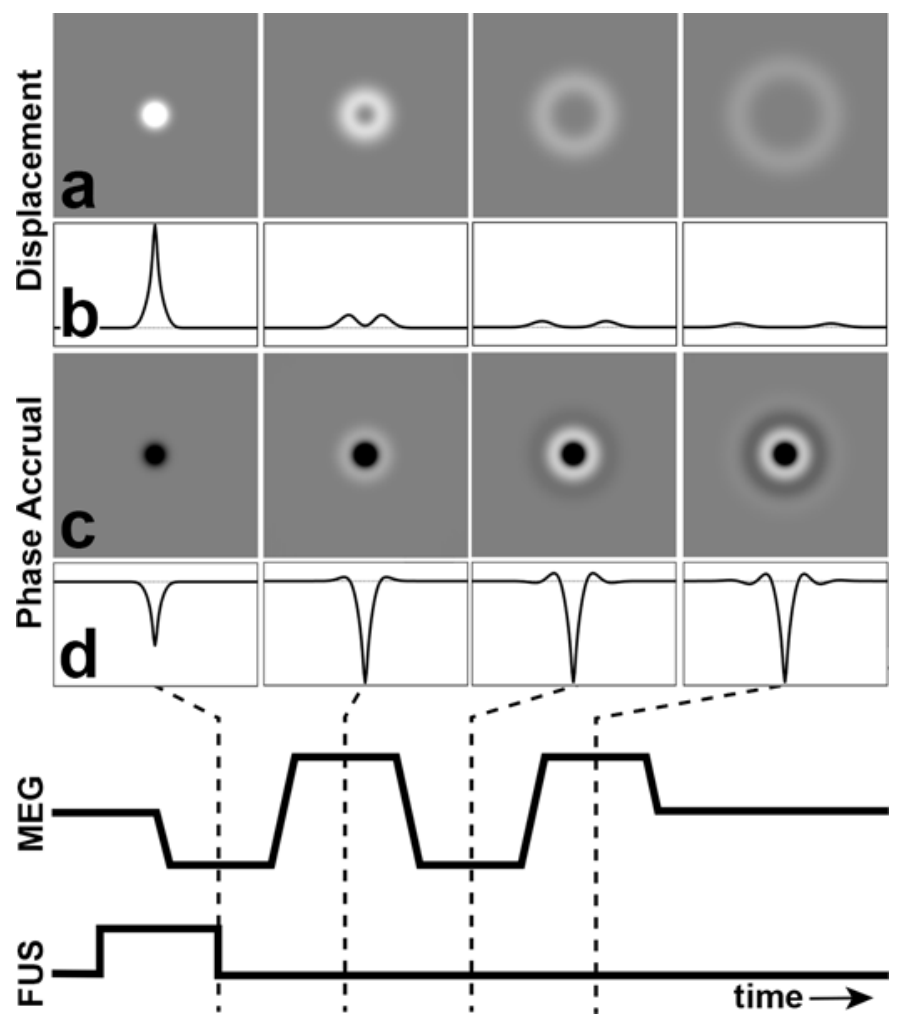

Figure 2.

Encoding of transient shear wavefront (rows a, b) in MR image phase (rows c, d) of a coronal imaging slice at isocenter with a short MEG train oriented in the thru-slice direction. Row (b) and (d) are horizontal line plots through the center of images (a) and (c), respectively, and depict the displacement and accrued phase profiles for each simulated time-point. Each maximum or minimum ring in the image phase encodes the location of the transient shear wave at sampling times denoted by the dotted lines. The MEG and FUS waveforms denote the relative timing of the applied ARF impulse and the MR pulse sequence motion encoding gradients. 

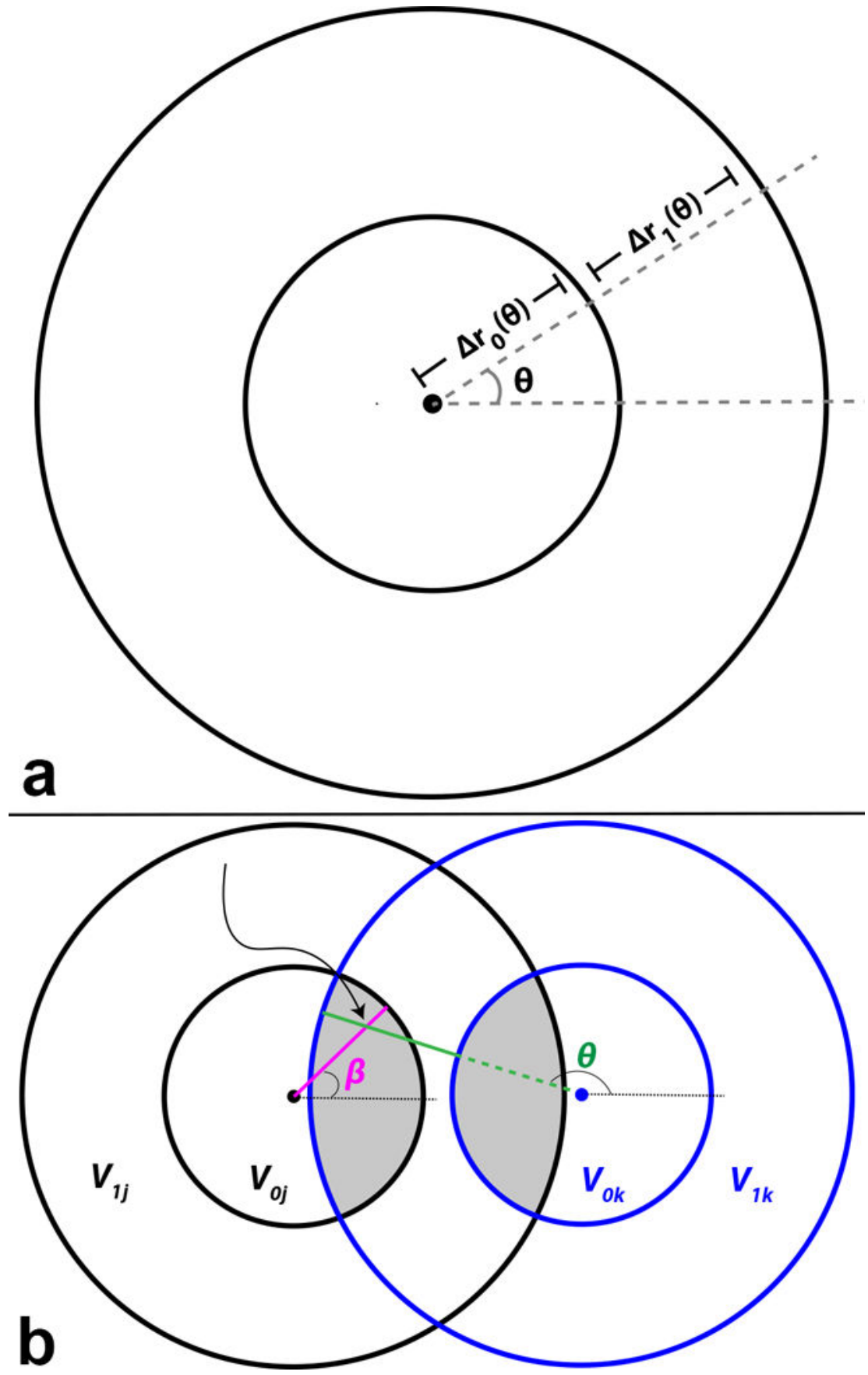

Figure 3.

A schematic of local extrema in the MR image phase is depicted in (a). The central dot denotes the initial ARF impulse location and the two black concentric circles denote the position of the encoded shear wavefront at two unique time-points. The distance (along a projection of angle $\theta$ ) between the ARF impulse location and first local extrema is denoted by $\Delta \mathrm{r}_{0}(\theta)$. The distance between this first and second extrema along the same projection is denoted by $\Delta \mathrm{r}_{1}(\theta)$. A schematic of the local extrema from two phase images acquired with different ARF impulse positions is shown in (b). $\mathrm{V}_{0 \mathrm{j}}$ and $\mathrm{V}_{1 \mathrm{j}}$ denote regions between 
adjacent encoded wavefronts from the $\mathrm{j}^{\text {th }}$ phase image. $\mathrm{V}_{0 \mathrm{k}}$ and $\mathrm{V}_{1 \mathrm{k}}$ denote these same regions for the $\mathrm{k}^{\text {th }}$ image. The overlapping gray shaded regions represent areas were $\mathrm{t}_{0} \mathrm{can}$ be calculated numerically. In principle, the number of peak/trough pairs is not limited to three and is determined by the MEG train length and the decay of the propagating shear wavefront amplitude. Only three position encodings per point are displayed here to simplify the diagram. 

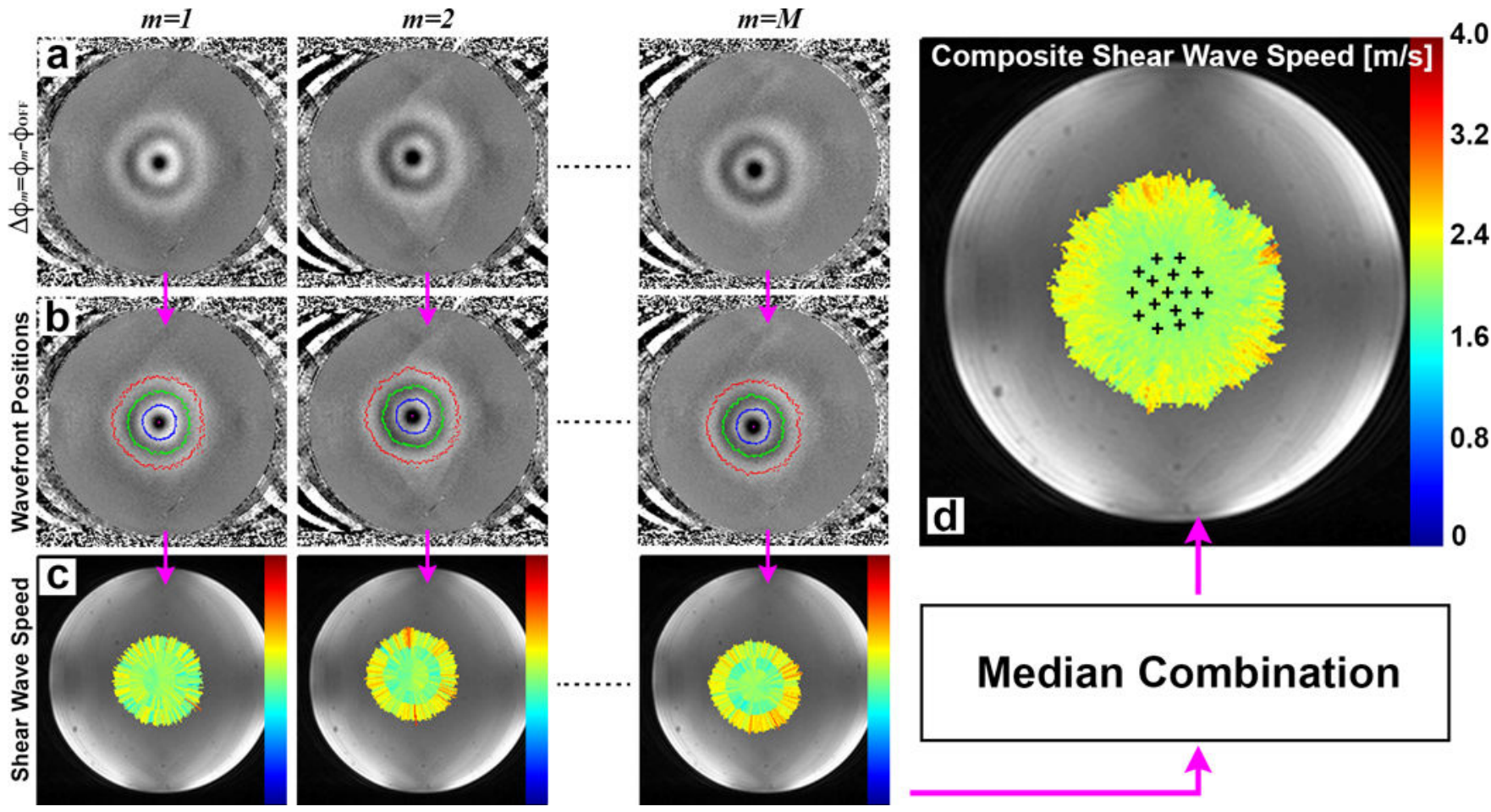

Figure 4.

MR-SWE shear wave speed calculation. MR phase difference images are depicted in row (a) where $\mathrm{M}$ denotes the number of phase images acquired with distinct ARF positions.

Wavefront locations, depicted as color overlays in row (b), are automatically extracted from phase difference maps using a template matching approach. The shear wave maps for each ARF point are calculated and shown in (c). The composite shear wave speed map (d) is generated by taking the median of measurements in pixels with 3 or more non-zero shear wave speed values. Crosses in (d) denote the location of the 16 unique ARF points. 


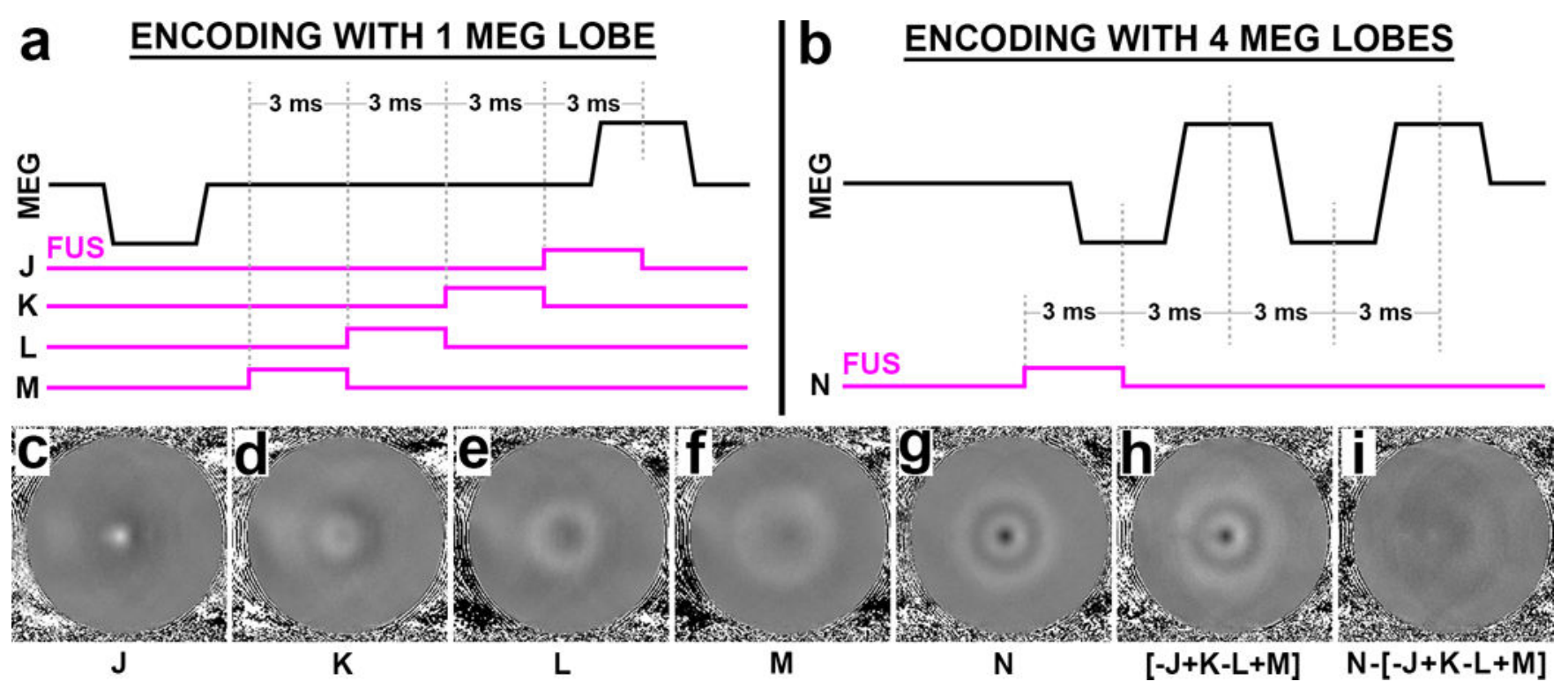

Figure 5.

Comparison of single lobe (a) and four-lobe (b) MEG encoding strategies. Letters J, K, L, and $\mathrm{M}$ are used to represent acquisition from single-lobed encoding where delay between start of 3 ms FUS pulse and center of the MEG lobe is 3, 6, 9, $12 \mathrm{~ms}$, respectively. Letter $\mathrm{N}$ is used to represent four-lobed acquisition where delay between start of 3 ms FUS pulse and center of first MEG lobe is $3 \mathrm{~ms}$. Phase images for the single lobe acquisition minus a reference phase image (no FUS) are depicted in (c-f). Phase image using the four-lobed acquisition minus a reference phase image (no FUS) is depicted in (g). A composite of images (c-f) by summing all images and alternating sign of adjacent pairs is shown in (h). This combination effectively shows that the four-lobed acquisition contains information encoded in the separate four single lobe measurements. The difference between $(\mathrm{g})$ and $(\mathrm{h})$ is shown in (i). 

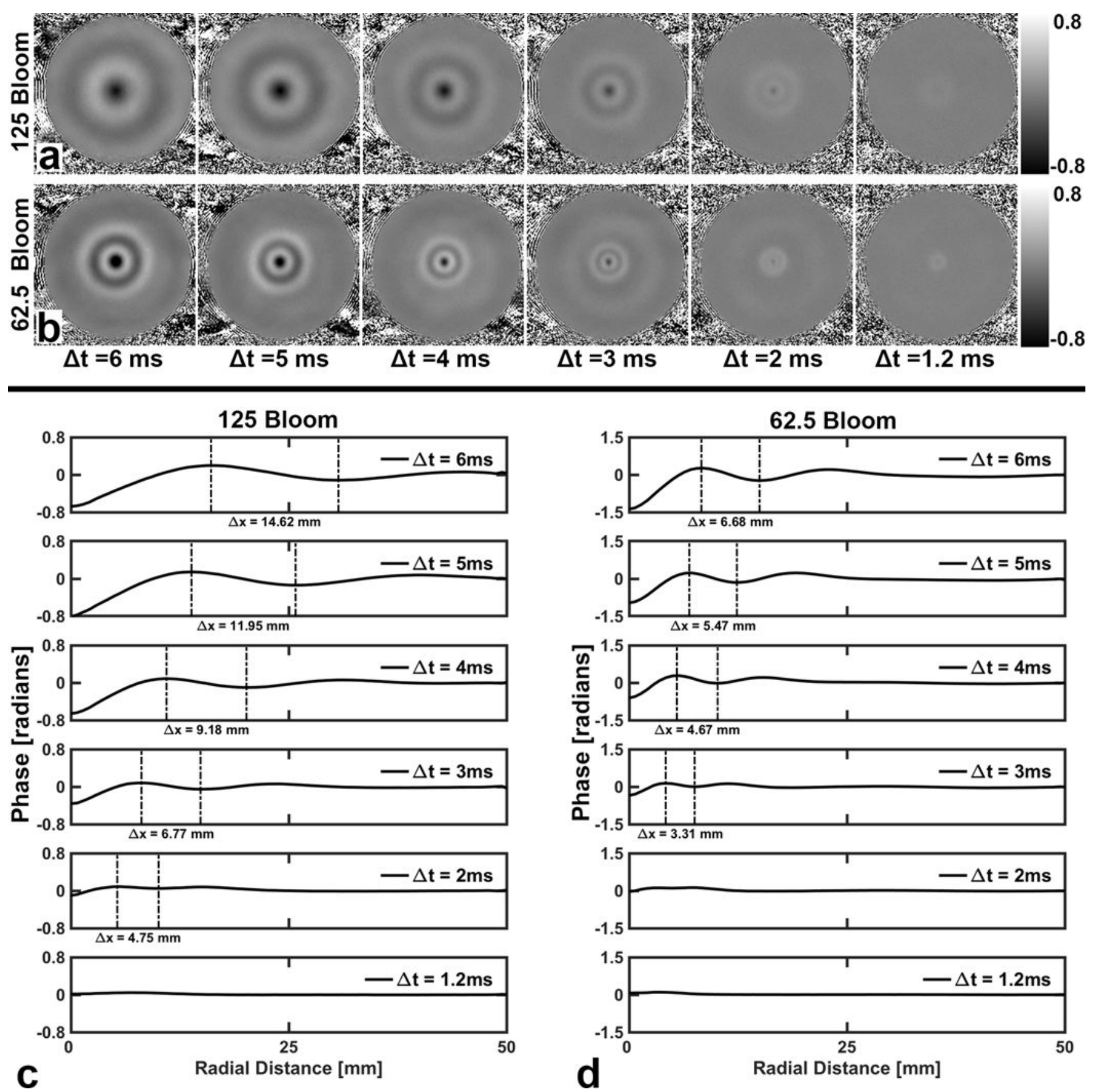

Figure 6.

Phase difference images of 125-bloom (row a) and 62.5-bloom (row b) phantoms where $\Delta \mathrm{t}$ denotes the spacing between adjacent MEG lobes. MEG lobes were sequential and each lobe duration was also equal to $\Delta$ t. For all images, a four-lobed MEG encoding was used. Images from (a) and (b) were converted to radial coordinates where the origin of the coordinate system corresponds to the central ARF location. All radial rays were averaged to generate the line plots shown in (c) for the 125-bloom phantom and (d) for the 62.5 bloom phantom. The vertical dotted lines in each figure denote the first local maximum and second local 
minimum in each line plot. The distance between these dotted lines provides a measure of the spacing between sampled shear wavefronts. For decreasing $\Delta t$ the spacing between encoded wavefronts decreases (finer resolution of sampled shear wavefront positions). Decreasing this distance results in finer resolution shear wave speed maps. However, the magnitude of the phase accrual also decreases with decreasing $\Delta t$, making it more difficult to detect the encoded wave front positions. 

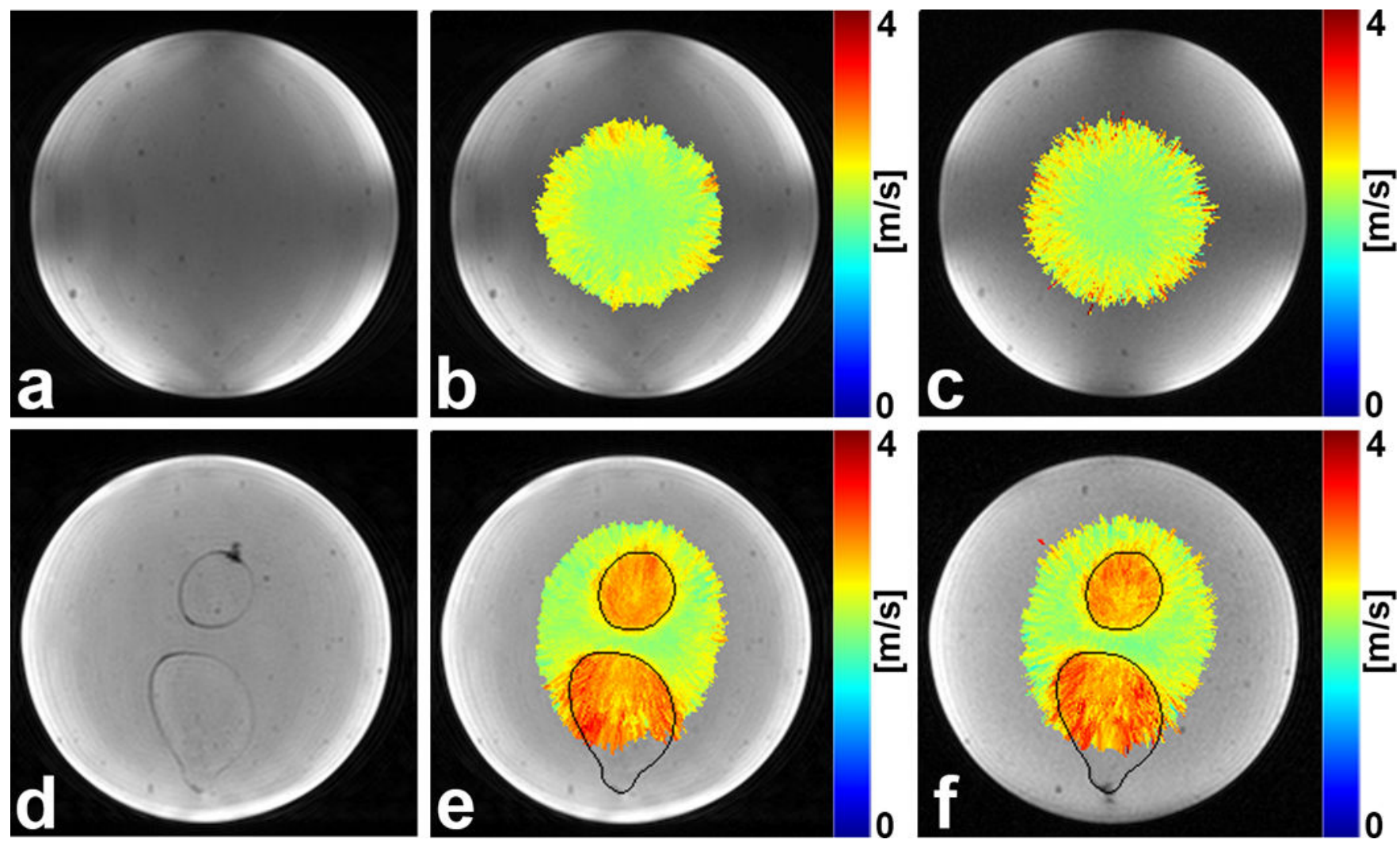

Figure 7.

MR-SWE shear wave speed measurements in homogeneous (a-c) and inclusion (d-f) phantom. Magnitude images are depicted in (a) and (d). MR-SWE results from 3D acquisition are shown in (b) and (e). Single slice (2D) MR-SWE acquisition results are shown in (c) and (f). Black borders in (e) and (f) denote the inclusion boundaries. 

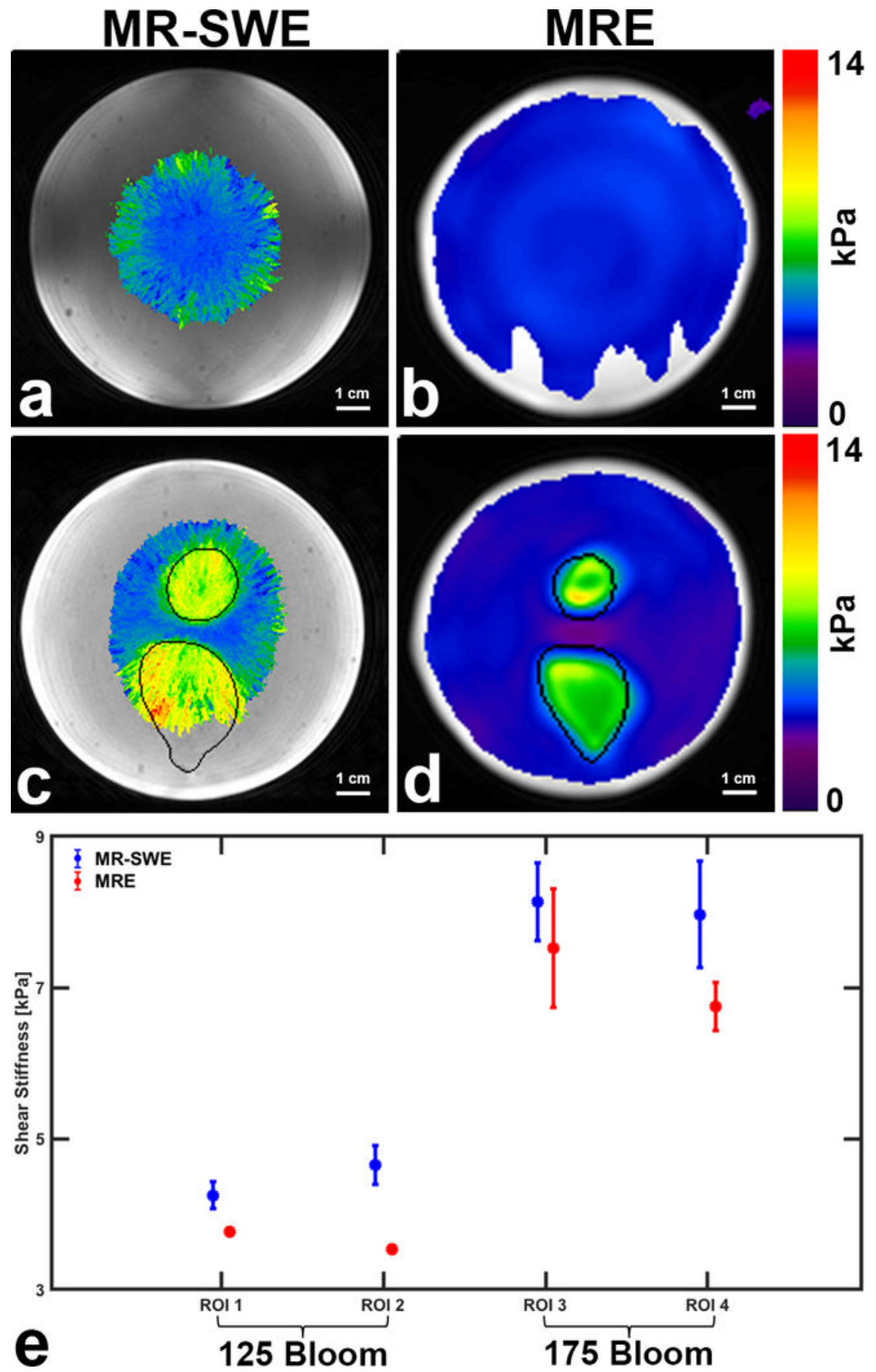

Figure 8.

Comparison of MR-SWE (a,c) and MRE (b,d) shear modulus maps in homogeneous $(\mathrm{a}, \mathrm{b})$ and inclusion (c,d) phantoms. Black overlays in (c) and (d) denote the inclusion borders. White scale bars correspond to $1 \mathrm{~cm}$. Mean and standard deviation of MR-SWE and MRE measurements in homogeneous (ROI 1) and inclusion (ROI 2-4) phantoms shown in (e) with ROIs ( 1 and 2) corresponding to 125 Bloom regions and ROIs ( 3 and 4) corresponding to 175 Bloom regions. In all ROIs, a two-sample t-test showed that MR-SWE and MRE measured values were statistically different $(\mathrm{p}<0.01)$. 

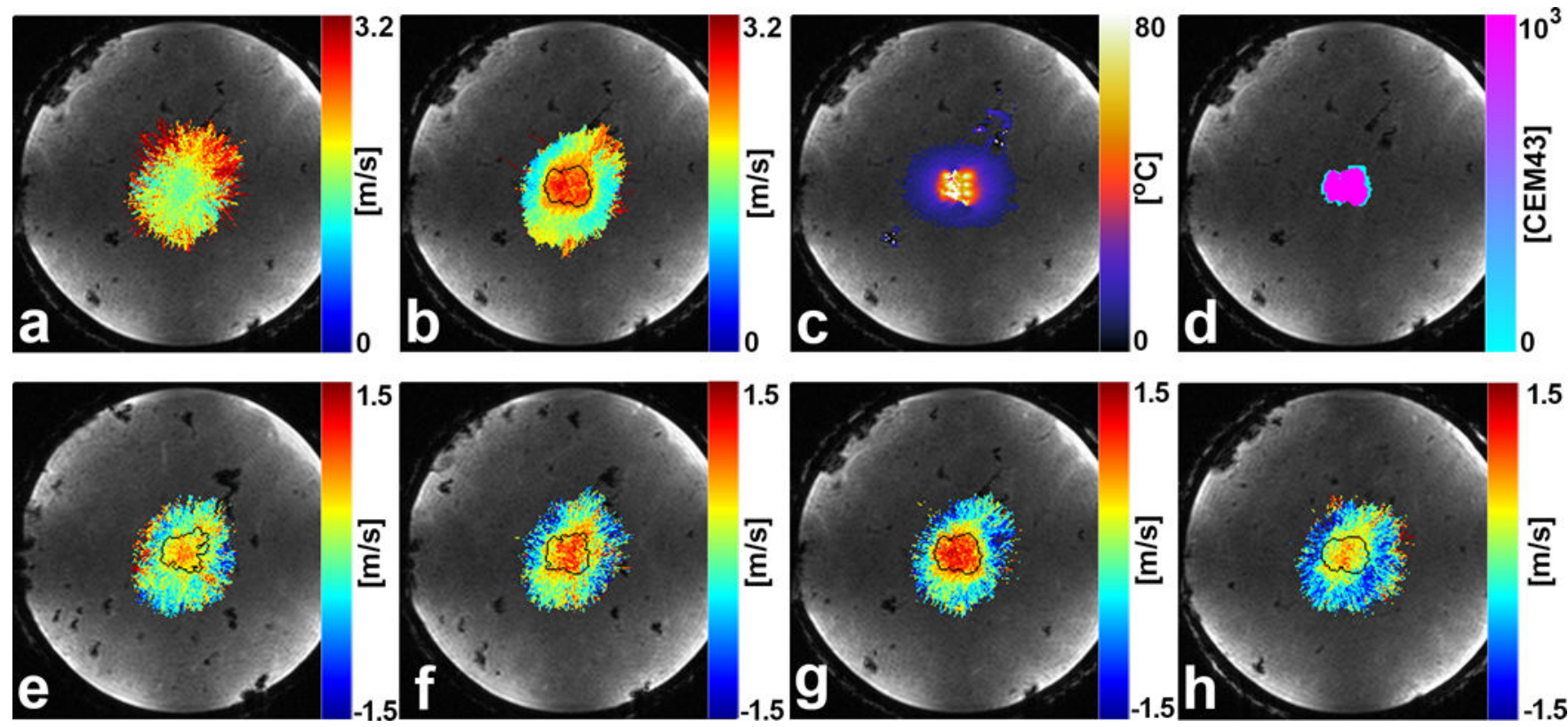

Figure 9.

Ex vivo ablation experiment. MR-SWE shear wave speed maps measured before (a) and after (b) ablation. Peak temperature change map (c) and calculated thermal dose (d) from ablation. The thermal dose threshold of 240 CEM43 is overlayed as a black line in (b). Area of increased shear wave speed is circumscribed by the 240 CEM43 thermal dose border. MR-SWE shear wave speed difference maps (post minus pre ablation) for 4 different slices (e-h). Black border overlay circumscribes the 240 CEM43 dose region. In all four slices, shear wave speed increased in the ablated zone. 


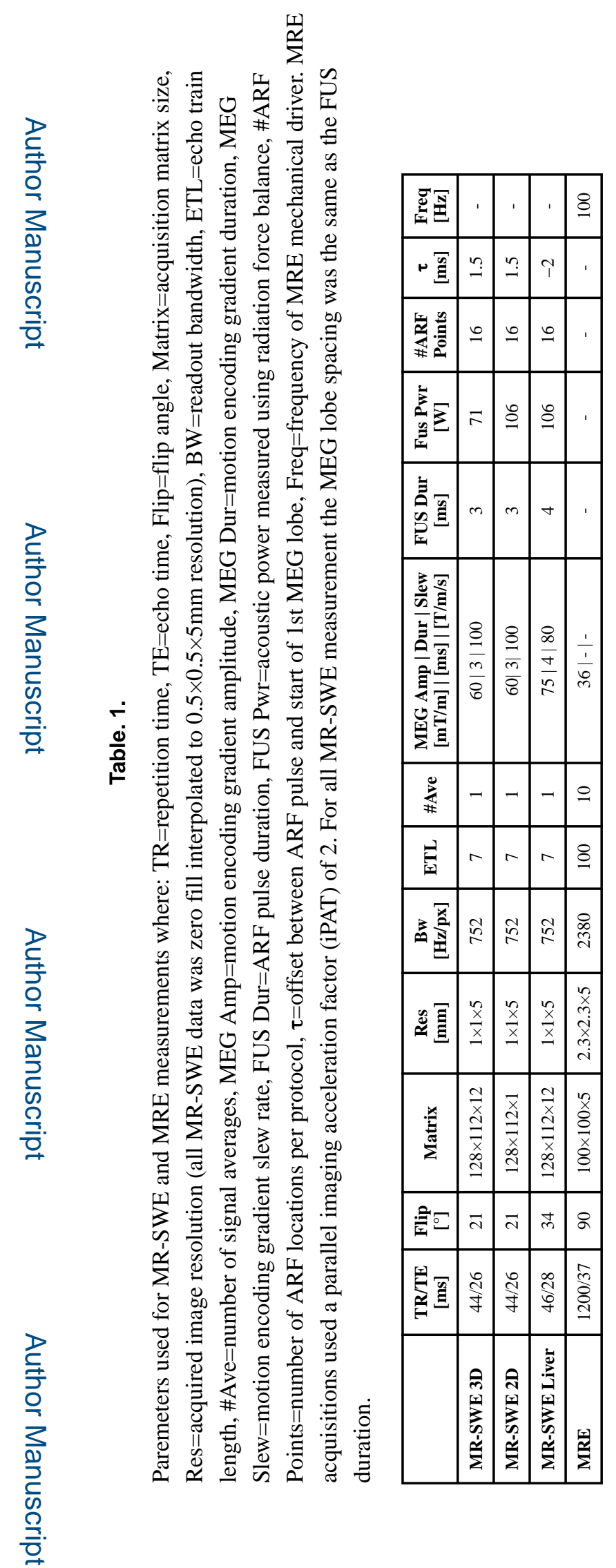

Magn Reson Med. Author manuscript; available in PMC 2020 May 01. 